\title{
FORMACIÓN INICIAL DEL PROFESORADO DE CIENCIAS EN FRANCIA, INGLATERRA Y GALES Y ESPAÑA. ANÁLISIS DE LA ORGANIZACIÓN DE LOS ESTUDIOS Y NUEVAS TENDENCIAS
}

\author{
DUMAS-CARÉ, A..$^{(1)}$, FURIÓ MAS, C. ${ }^{(2)}$ y GARRET, R. ${ }^{(3)}$ \\ (1) LIREPST. Université de Paris 7. \\ (2) Servei de Formació Permanent. Universitat de València. \\ (3) University of Bristol.
}

\section{SUMMARY}

This paper contains a comparative analysis of university courses on initial secondary teacher training in theses countries. The aim is to sort out the common components in these training systems. We also point out some contemporary trends in this field, trying to increase the degree of professionalisation of these courses.

\section{INTRODUCCIÓN}

El tema de la formación inicial del profesorado de secundaria es de plena actualidad no sólo en nuestro país, sino también en los de nuestro entomo europeo. Al haberse alcanzado cotas altas de escolarización obligatoria en los países avanzados, se plantea ahora como problema la mejora de la calidad de estas enseñanzas. Ello supone abordar, fundamentalmente, el tema de la formación del profesorado. Esta preocupación se puso de manifiesto en la conferencia de Ministros de Educación del Consejo de Europa, reunida en HeIsinki en 1987 , que acordo, entre otras, estas dos medidas urgentes (Imbernón 1990):

-La imagen de la enseñanza debe ser mejorada para permitir que a ella accedan candidatos valiosos.
-La formación del profesorado ha de ser concebida como un todo y considerada como una forma de educación permanente.

En general, esta formación del profesorado se entiende como un continuo con varias etapas que van desde la realización de los estudios de la formación inicial, pasando por un período de inducción durante el primero o los dos primeros años que el profesor ocupa su primer empleo, hasta la formación continua o permanente del profesor en activo con cierta experiencia docente (Bolam 1986). La primera de estas etapas es básica y decisiva en la futura formación permanente del profesorado de secundaria y, sin embargo, un informe reciente encargado por la Comisión de Educación de la C.E. ha puesto 
de relieve la falta de preparación profesional en estos estudios de formación inicial del profesorado de secundaria (Blackburn y Moisan 1986). En consecuencia, se está en período de revisión de los sistemas de preparación de este profesorado. Precisamente, con la finalidad de abrir perspectivas que puedan orientar futuros cambios, se presentan en este artículo los sistemas de organización de la formación inicial del profesorado de secundaria en Francia, Inglaterra y Gales y España. A continuación se establecen las oportunas comparaciones curriculares y se extraen algunas consecuencias que pueden ayudar a perfilar mejor la función profesionali. zadora de estos estudios.

\section{LOS SISTEMAS DE FORMACIÓN INI- CIAL DEL PROFESORADO DE CIENCIAS EN TRES PAISES EUROPEOS}

\subsection{La formación actual de profesores en Francia}

El sistema educativo francés descansa, básicamente, sobre tres tipos de estructuras: la enseñanza primaria (l'école élémentaire) de 7 a 11 años, el collège o enseñanza secundaria obligatoria con alumnos de 11 a 15 años (nivel II) y el lycée o enseñanza secundaria postobligatoria durante 3 años (nivel IIII). En el último de estos cursos (el terminaI) hay dos orientaciones propiamente científicas (el bac C, matemático y físico-químico y el bac $\mathrm{D}$, biológico y bioquímico), junto con otra más tecnológica (el bac E). La formación de los maestros de primaria (instituteurs) es diferente a la de los profesores de secundaria, si bien la reforma francesa de 1989 prevé un cambio importante que más adelante se comentará. En este trabajo sólo nos detendremos en la formación de los de secundaria, dado que en el nivel primario las Ciencias no son obligatorias y caso de enseñarse, sólo se

figura I

Estructura de la formación inicial del profesorado de secundaria en Francia

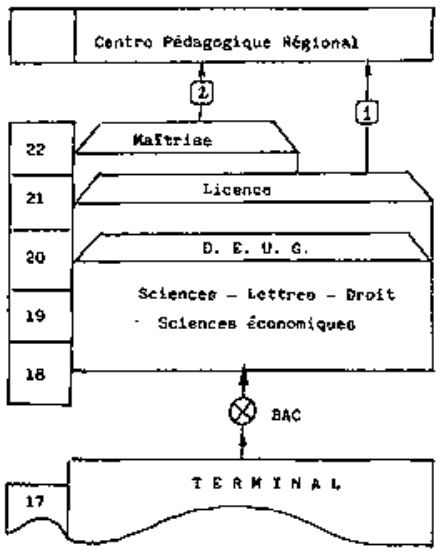

(2) $-5 x$. Agrçgation

(1) -Ex. C.A.P.E.S. presentan de forma opcional como actividades para despertar el interés de los alumnos (activités d'éveil).

En la figura I se han resumido las etapas en la formación de los dos tipos más frecuentes de profesores franceses que hay en las enseñanzas secundarias. Los futuros profesores de Física y Química (Sciences Physiques) acceden a través de dos concursos paralelos después de 3 y 4 años, respectivamente, de estudios universitarios especializados en una disciplina. Así pues, hay dos formas de acceso: el CAPES (Certificat d'Aptitude au Professorat de l'Enseignement Secondaire) para el que hace falta tener el título de diplomado ( 1 año de estudios además del DEUG) y la Agrégation -similar a la cátedra de Bachillerato- que se obtiene después del título de licenciado (2 años de estudios posteriores al DEUG). Los estudios de la diplomatura (license) y de la licenciatura (maîtrise) son sólo de contenidos científicos, pero desde hace algunos años, varias universidades han inciuido enseñanzas de Pedagogía y/o Didáctica destinadas a los futuros enseñantes. Por el momento, estos cursos no son obligatorios, pero hay una tendencia hacia su generalización en las universidades francesas.

Estos dos exámenes de acceso constan de una parte escrita y otra oral. Las pruebas escritas son esencialmente problemas y cuestiones de contenido conceptual de Física y Química. Las orales tienen más conexión con los problemas de la enseñanza, pero sólo se trata de desarrollar alguna lección o de hacer montajes de prácticas sobre el tema.

Una vez se ha superado el acceso, el futuro profesor asiste durante un año a un Centro Pedagógico Regional (CPR) donde concluye su formación. En este centro no universitario son impartidos por inspectores cursillos teóricos de Pedagogía, de Psicopedagogía y, a veces, de Didáctica especial (según el CPR). También hay una toma de contacto con el trabajo de aula bajo la supervisión de «consejeros pedagógicos», que suelen ser profesores de liceo con experiencia y que se han ofrecido voluntarios para formar a los jóvenes enseñantes. Al final de este año se realiza un examen de cualificación profesional y si se logra pasar se obtiene la acreditación de profesor por el candidato. El título obtenido se corres. ponde con el del examen que se aprobó en el acceso: agregé y certifié.

A partir de septiembre de 1990 , según establece la $L o i$ d'Orientation sur l'Éducation de 10 de julio de 1989 , se irán creando en cada Academia un Instituto Universitario de Formación de Profesores (IUFP), para maestros $y$ profesores de secundaria, adscrito a una o varias universidades. Así pues, con el propósito de integrar más estos estudios en la Universidad francesa funcionarán desde el curso 90-91 a título experimental Ios IUFP de Reims, Lille y Grenoble, donde se impartirán clases tanto por inspectores como por profesores de las propias universidades. En este proyecto se prevé que los aspirantes a profesores de enseñanza primaria y secundaria entren en el IUFP después de haber realizado 3 años de estudios en la Universidad. Estos estudios específicos en el IUFP durarán dos años y está en discusión si al terminarlos se obtendrá el título de licenciado en Cien- 
cias de la Educación para los profesores de primaria y el de licenciado en la materia específica para los de secundaria.

\subsection{Formación inicial de los profeso-res de Secun- daria en Inglaterra y Gales.}

El sistema de formación del profesorado en Inglaterra y Gales (Escocia e Irlanda del Norte tienen sistemas diferentes) es bastante distinto del francés. Tanto los profesores de primaria como los de secundaria pueden seguir indistintamente dos caminos para conseguir el estatus de profesor cualificado (Qualified Teacher Status) que habilita para enseñar : a) el Postgraduate Certificate in Education (PGCE) y b) el Bachelor of Education degree (BEd).

En la práctica la vía del PGCE es seguida mayoritariamente por los futuros profesores de secundaria obligatoria (de 11 a 16 años) o postobligatoria (16-18 años, sixthform). Primero se obtiene una licenciatura en una materia durante 364 años de estudios (Bachelor of Arts o Bachelor of Science) en una Universidad, un Politécnico o un College of (Higher) Education y después se sigue un curso de postgrado de estudios profesionales (el PGCE) en uno de aquellos centros (fig. 2).

\section{figura 2}

Estructura de la formación del profesorado de secundaria en Inglaterra y Pais de Gales.

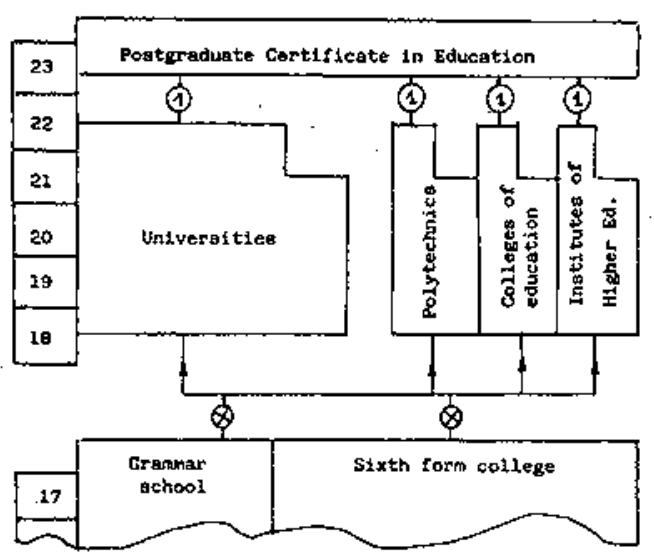

(1) -B.SC. 0 $-B . A$.

El grado de Bachelor of Education es la cartera destinada a la formación del profesorado de enseñanza primaria, dividida en dos especialidades (de 3 a 8 años, early years of schooling, y de 7 a 12 años, Junior), de las que no nos ocuparemos en este trabajo (Blasco 1989).

En la actualidad y debido, en particular, a la falta de profesores de secundaria en algunas materias científicas
(Matemáticas, Física, Química y Tecnología) se están ensayando nuevos sistemas de formación más cortos como el licensed teacher o el articled teacher. Estas vías pretenden atraer graduados en Ciencias que trabajan en la industria o en el comercio, aunque no hayan obtenido el QTS. Las condiciones mínimas que tiene que cumplir el candidato para acceder a licensed teacher son: a) ha. ber obtenido una nota $E$ en Inglés y Matemáticas en el examen del General Certificate of Secondary Education (que se realiza al final de la secundaria obligatoria), b) haber cursado un mínimo de dos años o equivalentes de estudios universitarios, y c) tener por lo menos 25 años de edad (a excepción de los profesores formados en países anglófonos de ultramar de la Commowealth). Este futuro profesor se adscribe a una escuela donde se diagnostican sus necesidades de formación, se le nombra un tutor, experto en el area de la materia que desea enseñar, se planifica su formación en el período de license y finalmente, después de realizado, se le evalúa de acuerdo con una serie de conocimientos y destrezas que debe haber adquirido. La responsabilidad de la formación de este profesor recae en la autoridad educativa local de la que depende administrativamente la escuela donde se le adscribió.

En cuanto a la organización académica del PGCE, se puede indicar que, en general, tiene 3 grandes componentes curriculares:

a) Subject Method Studies que corresponden a los estudios de la didáctica de la materia principal que se ha estudiado en la licenciatura (p.e. The Chemistry Course). También se suelen of recer estudios de una disciplina complementaria. Estos cursos tienen una vertiente eminentemente práctica, pues desde ellos se prepara la estancia de los alumnos en las escuelas.

b) Educational Studies, centrados en la componente psico-socio-pedagógica general de estos estudios y donde se ofrecen un conjunto de cursos cortos como puede ser el tratamiento de los factores sociológicos, pedagógicos y psicológicos del proceso de enseñanza-aprendizaje, tutorías, alumnos con necesidades especiales o problemas educativos de importancia, como las tendencias del currículo, evaluación y exámenes, educación y sexo...

c) Las prácticas de enseñanza (School experience) comprenden la mitad del tiempo de las 36 semanas que viene a durar el curso y se realizan de forma progresiva a lo largo de los 3 trimestres del curso.

La oferta de cursillos en las componentes a) y b) es variable y depende de las universidades (autónomas), de los politécnicos y colleges (controlados por las autoridades locales). Pero, esta descentralización tradicional del sistema educativo inglés tiende actualmente hacia una cierta uniformización. En efecto, siguiendo las recomendaciones del Advisory Comittee on the Supley and Education of Teachers (DES 1983), el Departamento de Educación y Ciencia mediante una circular (DES 1984) ha establecido un mecanismo para la homologación de los cursos de formación inicial de profesores que realizan las instituciones inglesas encargadas de esta labor. 
Esta homologación fue definida por un Consejo ad hoc (Council for the Accreditation of Teacher Education, CATE 1985) en base a un conjunto de criterios sobre:

-la selección y admisión a los cursos de formación inicial de profesores

-las relaciones entre las instituciones de formación y las escuelas

-el tiempo mínimo de estudios universitarios de la materia a enseñar y su atención a la especialización didáctica

-los estudios psicopedagógicos generales y las prácticas profesionales.

\section{3. La formación inicial del profesorado en Cien- cias en España}

Aunque, en teoría, nuestro sistema de formación inicial del profesorado de Enseñanzas Medias se asemeja en su estructura al inglés, en la práctica las diferencias son muy notables. A partir de 1970 se crea los Institutos de Ciencias de la Educación en cada universidad española con la finalidad, entre otras, de ofrecer a los titulados superiores (licenciados, ingenieros y arquitectos) que desearan ser profesores un Curso de Aptitud Pedagógica (CAP) de 1 año de duración (más concretamente 300 horas). Se entendía que la formación inicial de este profesorado debía consistir en la suma de dos componentes: la componente básica o específica de la materia que se había cursado en la licenciatura (en general, 5 años) y la componente pedagógica teórico-práctica (CAP) que estaría destinada a aportar la formación profesionalizadora. El CAP legalmente consta de dos ciclos, el primero de ellos con 150 horas para impartir enseñanzas teóricas de Sociología, Psicología, Pedagogía, Didáctica General y Didácticas especiales, y el segundo ciclo, también con 150 horas, destinado a las prácticas de enseñanza en un centro de enseñanza media a cargo de profesores tutores con experiencia docente.

En la práctica, la aplicación de este sistema de formación de los profesores de secundaria ha sido variable y ha dependido de cada ICE. Pero, globalmente, se puede afirmar, que la experiencia ha sido valorada negativamente debido, entre otras razones, a que es vivida por los estudiantes del último año de carrera como simple requisito burocrático para enseñar, y como estudios adicionales sin interés en comparación con sus estudios ordinarios (Furí6 y Gil 1985). Como bien indica el Grupo XV, comisionado por el Consejo de Universidades para proponer una nueva formación del profesorado de secundaria (1989), el sistema actual carece de estructura estable (ni siquiera dispone de plantilla de profesorado), de recursos personales y materiales y es de escasa calidad profesionalizadora.

Un breve estudio cronológico de este problema puede explicarnos los desfases reales existentes entre el sistema inglés y el español. En España y desde la creación a partir de la Ley Moyano (1857) de las Facultades de Ciencias Exactas, Físicas y Naturales que se separaron de las de Filosofía y Letras, sólo había que licenciarse para ser profesor de Ciencias en un Instituto (Moreno 1990). Es en los albores del s.XX cuando en los Congresos de Pedagogía comienzan a aparecer menciones explícitas a la componente pedagógica en la formación del profesorado de EEMM. Así en 1892, el Congreso Pedagógico Hispano-Portugués-Americano(Madrid), en su séptima conclusión, propone la creación de un Centro Pedagógico en el que puedan adquirir conocimientos de esta índole los aspirantes a aquel profesorado. Es más, en 1900el Congreso de Enseñanza Superior de París (Gutiérrez 1980) cita literalmente que céstos (los profesores de EEMM) reciban una formación pedagogica teórica y práctica, mediante la historia de la pedagogía, la disclisión de los métodos, y los ejercicios profesionales de aplicación. Esta conclusión es asumida por los representantes de diversos países europeos y americanos y, entre ellos, se encontraban los de Gran Bretaña, Francia y España. Con anterioridad el informe de la Cross Commision inglesa (Royal Commision 1888) había instado a las universidades a implicarse en la formación de los profesores de las escuelas, pero es a partir de 1902, cuando se institucionaliza la formación del profesorado de secundaria según la Educational Act del mismo año (Patrick et al. 1982) y en esta época empiezan a generalizarse estos estudios en los Colleges de Educación. En cambio, el primer precedente legal sobre la introducción de la componente pedagógica en la formación de profesores de secundaria la encontramos en el primer Boletín de Educación del Ministerio de Instrucción Pública de la II República española (1933) donde se manifiesta que «Las Facultades de Fílosofía y Letras y de Ciencias incluirán en sus enseñanzas como obligatorias para los opositores, cursos teorico-prácticos de carácter esencialmente pedagógico, a fin de que los aspirantes al profesorado hayan podido realizar estudios de Pedagogía General y Metodología» (Moreno 1990). Habrán de pasar algunas décadas más para que la Universidad empiece a plantearse el tema con la creación de las Escuelas de Formación de Profesorado en la década de Ios 60. Pero, es en 1970 cuando se crean los ICE con competencias legales sobre la formación inicial del profesorado de secundaria, si bien en la práctica queda por resolver el problema de una institucionalización real de estos estudios. En la actualidad en España se están reformando todos los subsistemas educativos y, entre ellos, el de la formación inicial de los profesores de secundaria. La propuesta emitida por la ponencia que sobre este tema ha creado el Consejo de Universidades consiste, básicamente, en un curso de postgrado de 600 horas estructurado en base a los siguientes contenidos: Sociología de la Educación, Psicología del desarrollo, Psicología de la Educación, Teoría de la Educación, Didáctica general, Didáctica especial, Organización escolar, Problemas de aprendizaje y adaptaciones curriculares, Contenidos sustantivos complementarios (según la especialidad o área) y Practicum. Es, pues, oportuno y necesario abrir este debate para buscar soluciones al problema de la formación inicial del futuro profesorado que deber enseñar Ciencias a adolescentes y estudiantes de edades comprendidas entre 12. y 18 años. 


\section{CONSIDERACIONES SOBRE LA OR- GANIZACION CURRICULAR DE ESTOS ESTUDIOS}

Expuestos los sistemas de formación inicial del profesorado de estos tres países, pasaremos a continuación a hacer algunas consideraciones extraídas del análisis de sus componentes curriculares y de algunos resultados de la investigación en la enseñanza de las Ciencias.

Es bien sabido que el grado con que se interrelacionan la teoría y la práctica en esta formación profesional es uno de los indicadores más importante de la calidad de la misma. Atendiendo a esta dimensión teoría-práctica, normalmente se distinguen en el currículo de la formación de profesores tres componentes:

\section{a) La componente científica oformación básica sobre la ciencia que se va a enseñar.}

b) La componente profesional práctica que pretende dar un conocimiento del contexto didáctico y que según algunos autores define el mayor o menor grado de profesionalización adquirido en las experiencias de campo del sistema de formación inicial (Blackburn y Moisan 1986).

c) La componente profesional académica que contiene un conjunto de saberes o conocimientos sobre los alumnos de un determinado nivel educativo y que, por lo general, vienen en forma de disciplinas de Ciencias Sociales.

Analicemos con un poco más de detalle estas tres componentes curriculares con el fin de proponer algunas orientaciones que puedan mejorar la organización de estos estudios.

\subsection{La formación básica de los futuros profesores en la disciplina que tienen que enseñar es incuestionable}

Una de las competencias que se consideran fundamentales en el perfil de un profesor de Ciencias es el dominio de los contenidos que se van a impartir. En efecto, es aceptada por todos incluidos aquéllos que ingenuamente parten del supuesto que ensefiar una disciplina es fácil y que sólo requiere conocimientos de la materia a enseñar y un poco de «sentido común». Así está contemplado en los sistemas de organización de los estudios de formación inicial del profesorado de secundaria en los tres países presentados en el apartado anterior. En efecto, en Francia se observa que hay una exigencia de, al menos, 3 ó 4 años de estudios de la disciplina (eI DEUG más 1 o 2 años) según se acceda con el título de licence o de mattrise, respectivamente. En Inglaterra y Gales ya se ha indicado que tradicionalmente los futuros profesores de Ciencia de secundaria acceden al PGCE con el Bachelor of Science y ello significa prácticamente 364 años de contenidos de la disciplina científica principal. En España, por lo general, se requieren 4 ó 5 años para poder cursar el CAP, según se encuentren en el último año de carrera o sean licenciados, respectivamente.

De hecho conocer en profundidad una disciplina implica saber no sólo de manera estética el conjunto actual de conceptos, principios y teorías que la constituyen, sino también cuál ha sido la dinámica seguida en la construcción de dichos conocimientos, qué barreras epistemológicas han tenido que vencer las comunidades científicas, cuáles son los problemas no resueltos, qué características son esenciales en las metodologías o procedimientos empleados, etc... Estos saberes serán fundamentales para que el profesor pueda seleccionar los conceptos y procedimientos fundamentales del cuerpo de conocimientos que quiere transmitir, pueda plantear sifuaciones problemáticas en el desarrollo de la asignatura y, en definitiva, pueda ejercer una enseñanza eficaz. Los resuitados de investigación a este respecto muestran que este conocimiento de la materia es la primera condición para poder realizar una enseñanza efectiva como se destaca en encuestas hechas entre los propios profesores de Ciencias (Duarte y Fontes 1989) e incluso se resalta que el desconocimiento por el profesor de los contenidos supone de facto una grave dificultad en la mejora cualitativa del profesor (Tobin y Espinet 1989), y un verdadero obstáculo al cambio didáctico (Hewson y Hewson 1987 y 1988).

No obstante, debido a la necesidad social de atender el aumento de escolarización en la enser̃anza secundaria y, en consecuencia, de profesores titulares de las asignaturas científicas que se está dando actualmente en los países en cuestión, se va hacia una disminución de la duración de esta componente básica. Así está ocurriendo ya en las nuevas modalidades inglesas de licensed y articled teachers, como se puede advertir en el criterio establecido por el CATE para esta componente formativa: un mínimo de dos años de estudios universitarios en la disciplina de la que se desea ser profesor. En Francia también se ha observado un menor número de candidatos que plazas de Ciencias ofertadas a nivel nacional durante los últimos concursos anuales del CAPES y de la Agrégation. Así pues, habrá que buscar soluciones a este problema de la formación inicial del profesorado de Ciencias que pasa necesariamente por idear alternativas con esta componente básica más corta. También creemos que sucederáa pronto en España y por ello sería conveniente y urgente el establecimiento de una doble vía para la formación de este profesorado. Una vía corta consistiría en la integración de la formación profesional docente, como especialización en el segundo ciclo de los estudios universitarios de la licenciatura, para aquellos estudiantes que ya tuvieran decidida su incorporación a la docencia. Ésta es la opción más valorada tanto por alumnos como por profesores del CAP en un estudio realizado en Ia Universidad de Valencia (Furió, Gil y Senent 1988). La segunda opción estaría organizada como estudios de postgrado para aquellos licenciados que también quieran especializarse como profesores y hayan cursado otras especialidades. Estas dos opciones fueron recomendadas recientemente por el Consejo Escolar del Estado español (1990) en sus sesiones de marzo, dentro del dictamen que preceptivamente elevó al Gobiemo sobre el anteproyecto de la Ley de Ordenación General del Sistema Educativo. 


\subsection{Las prácticas de enseñanza: elemento profesio- nalizador de la formación inicial del profesorado de Ciencias}

En el apartado anterior se ha indicado que la componente básica del currículo de la formación de profesores de Ciencias tiene por objeto desarrollar en el futuro profesor sus conocimientos respecto a la disciplina. Otra de las componentes esenciales en la organización de esta preparación es el período de prácticas, ya que se considera relevante la vivencia directa y personal de los procesos informales que se desarrollan en estas experiencias de campo. Adquirirán así los futuros profesores las bases del conocimiento del contexto didáctico en el que se desarrollan estas enseñanzas. Estas prácticas, debidamente orientadas, planifícadas, realizadas y analizadas, constituyen, sin duda, una verdadera iniciación a la profesión docente, de ahí que se tomen como elemento clave profesionalizador del currículo.

En efecto, Schön (1983) llama la atención sobre este elemento de la formación profesional docente y comenta críticamente que durante demasiado tiempo la preparación inicial de los profesores se ha venido basando en un modelo de «racionalidad técnica», donde implícitamente se admite que el conocimiento profesional se deriva de la aplicación de las ciencias académicas, cuando, según este autor, la práctica de esta profesión y de otras similares como la medicina, la ingeniería o la arquitectura se basa en un conocimiento distinto que denomina «conocimiento en acción». En el mismo sentido, Zeichner (1983), tomando en consideración dos dimensiones, define la existencia de cuatro paradigmas de formación del profesorado y, dentro de ellos, defiende el centrado en la investigación crítica y la reflexión sobre las causas y consecuencias de las acciones de clase, de manera que el futuro profesor pueda adquirir cierta capacidad intelectual al valorar su propia práctica.

En resumen, las prácticas de enseñanza han sido enfatizadas por esta línea de investigación basada en el estudio de cómo aprenden los profesores a enseñar, y en el análisis de su proceso de pensamiento y de la correspondiente toma de decisiones cuando se interaccionan en las clases. Estas prácticas serán el medio idóneo para llevar a cabo una enseñanza reflexiva y constituir un elemento fundamental en la formación del futuro profesor, ya que le capacitará para valorar de forma crítica su práctica y, al propio tiempo, se le inicia en la investigación-acción (Calderhead 1989).

Este énfasis en la componente práctica del currículo se aprecia en los sistemas de formación inicial del profesorado de Ciencias que se han presentado. En el sistema francés se observa que durante el año que el futuro profesor asiste a un Centro Pedagógico Regional tiene programadas unas prácticas en las que se le asigna un consejero pedagógico, como profesor tutor con experiencia que le guiará en estas prácticas. El peso de estas prácticas de enseñanza en el PGCE inglés es, aproximadamente, el $50 \%$ de las 36 semanas que dura este postgrado. Es más, dentro de los criterios actuales expuestos por el CATE se explicita que el período de estas experiencias de campo no puede ser inferior a 15 semanas en el PGCE o a 20 semanas en el BEd de 4 años. En el caso español estas prácticas que se fijaron legalmente en 150 horas, en la realidad son mucho más breves đebido en particular a la poca disponibilidad de la mayor parte de los estudiantes que están cursando, al mismo tiempo, su último año de carrera. Esta debilidad de la componente práctica del CAP es una característica ampliamente reconocida (Furió y Gil 1985, Imbernón 1989, informe del Grupo XV del Consejo de Universidades). Pero, a pesar del corto período de tiempo que duran estas prácticas en el caso español, se ha comprobado durante varios años consecutivos que esta componente es la mejor valorada por los futuros profesores, independientemente de la carrera cursada (Furió, Gil y Senent 1988).

\subsection{La componente profesional académica: hacia un modelo de formación del profesorado de Ciencias que integre la teoría y la práctica}

En el apartado anterior se ha puesto de relieve la necesidad de las prácticas de enseñanza en el currículo de la formación profesional del docente, pero ello no debe significar situarse en uno de los extremos del continuo teoría-práctica. Decíamos que un buen programa de estas prácticas de enseñanza requiere una orientación y planificación adecuadas. Es decir, requiere un marco teórico que las guíe; de lo contrario, nos encontraríamos en el denominado y criticado por el propio Zeichner (1983) como paradigma tradicional-artesanal, donde se concibe la formación del profesor como un proceso de modelización en el que el futuro profesor mediante ensayo y error aprenderá a imitar al profesor tutor de sus prácticas de enseñanza.

Este marco teórico que, en general, viene en forma de diversas materias de Ciencias sociales en los distintos currículos formativos del profesorado vendría a proporcionar al estudiante unos conocimientos sobre las características de los alumnos de un nivel concreto, sobre la organización escolar del mismo y sobre los problemas docentes relativos a facilitar la construcción de conocimientos por el conjunto de la clase a cargo del profesor. Se concibe así que las ideas aportadas por aquellas disciplinas pueden actuar como sistema de referencia a la hora de preparar las prácticas y, también, les proporcionará elementos críticos sobre las mismas.

Ahora bien, al abordar la composición curricular de esta formación profesional académica se suele adoptar un modelo sumativo de las diferentes disciplinas de Ciencias sociales que tienen algo que ver con la acción educativa. Así vemos como a principios de este siglo se introdujo la componente pedagógica en esta formación del profesorado de secundaria, abarcando con esta denominación todos aquellos aspectos complementarios a la formación básica (licenciatura). Dentro de ella estaba comprendida la didáctica general referida al estudio de los métodos de enseñanza y al análisis de los recursos didácticos propios de un nivel de enseñanza. Más adelante, se defiende la inclusión de aquellas disciplinas más directamente ligadas a la formación de la personali- 
dad del educandoen desarrollo y a los factores sociológicos del aprendizaje (Conant 1963). Surgen así las componentes psicológica y sociológica de la educación.

En consecuencia, asumir este modelo supone hacer una lectura horizontal de los distintos saberes teóricos que se necesitan para afrontar el problema, en nuestro caso, de enseñar una disciplina científica en un contexto didáctico dado. Precisamente uno de los principales problemas que se han detectado en la formación del profesorado no es tanto el desarrollo de los conocimientos sobre los alumnos y las clases, cuanto que facilitar a los profesores en formación que integren aquellos conocimientos dentro de su propia práctica (Calderhead 1986). De ahí que uno de los principales indicadores de la calidad de la formación de profesores está relacionada con el grado de integración entre esta formación académica y la práctica profesional.

A este modelo sumativo se debe oponer una formación profesional académica más integradora en la relación teoría-práctica. En el nivel de la enseñanza secundaria, es preciso que la formación del profesorado de Ciencias se focalice más hacia los problemas de la enseñanza de los contenidos específicos que hacia aspectos más generales de la educación. Este papel de disciplina integradora puede y debe ser desempeñado por la didáctica especial, ya que puede actuar de interfase entre la disciplina científica y aquel conjunto de saberes psico-socio-peda. gogicos generales que hay que aplicar en su enseñanza. En efecto, plantearse con los futuros profesores de Ciencias la selección de unos contenidos científicos concre . tos, la dosificación en la introducción de los conceptos en clase, el diseño y la realización de problemas y trabajos prácticos que pretendan familiarizar a los estudiantes con las características procedimentales de las Ciencias y, en definitiva, enseñar a presentar la disciplina en forma de problemas a plantear y resolver de forma crítica en una perspectiva de cambio conceptual, metodológico y actítudinal, requiere tener en cuenta tanto los avances psico-pedagógicos como los resultados de la investigación didáctica. Y todo ello son problemas de estudio e investigación que se están abordando desde el cuerpo de conocimientos de la didáctica de las Ciencias (Tiberghien 1985, Furió y Gil 1989). Esta tendencia a considerar la formación inicial del profesorado como surna de una formación científica y unos complementos pisco-pedagógicos generales está siendo superada con la introducción de la didáctica especial que puede integrar los aspectos más teóricos con los prácticos (Pessoa de Carvaiho y Vianna 1988). En este sentido se comprende la existencia del bloque titulado Subject method studies en la organización curricular inglesa de esta formación y también que desde aquél se dirijan las prácticas de enseñanza de los futuros profesores de Ciencias. Repe. timos que ello no debe suponer rechazo o desconexión con materias como la Psicología, la Pedagogía, la His. toria de las Ciencias o la Epistemología que, sin duda, están haciendo aportaciones de interés al aprendizaje de las Ciencias, pero que serán integradas en el cuerpo de conocimientos de la Didáctica de las Ciencias.

\section{A MODO DE CONCLUSIÓN: LA FOR- MACION INICIAL DEL PROFESORADO REQUIERE EL IMPULSO DE LA INVES- TIGACION UNIVERSITARIA EN LA DI- DÁC'TICA DE LAS CIENCIAS}

En definitiva, una formación del profesorado de Ciencias en la enseñanza secundaria que vaya más allá de una concepción basada exclusivamente en una formación estrictamente científica (Piaget 1969), o de aquella otra pensada como adición simple de unos complementos psicopedagógicos generales a la científica, requiere su conversión en actividad netamente universitaria, al contrario de lo que habitualmente ha sido, y que su docencia se desarrolle en intima conexión con las aportaciones de la investigación en didáctica de las Ciencias. Tanto la formación inicial como la continua del profesorado no puede mantenerse al margen de la actividad investigadora, ya que como señalan Verma y Beard (1981) los profesores han de ser los principales consumidores de los hallazgos de la investigación educativa con el fin de poder examinar críticamente su trabajo en el aula. Es decir, «mejorar significativamente la docencia implica crear una tradición de investigación accesible a los profesores que nutra su docencia» (Stenhouse 1975) y para ello es necesario que en su primera formación ya se les inicie en esta tradición si realmente se desea que, después, cuando ejerzan como profesores se aproximen, al menos, a los resultados de la investigación (Lawson, Costenson y Cisneros 1986). De ahí la necesidad de que se impulse decididamente la investigación universitaria en la Didáctica de Ias Ciencias, ya que sus resultados serán el sustrato básico no sólo para los cursos de las disciplinas incluidas en la componente profesional académica, sino también en la formación continua y en el desarrollo de proyectos de innovación curricular que puedan suponer una transformación real de la enseñanza-aprendizaje de las Ciencias (Penick y Yager 1986).

Esta institucionalización universitaria de la actividad docente e investigadora centrada en la Didáctica de las Ciencias está llamada a desempeñar un papel relevante en la mejora de la organización de los estudios de formación del profesorado de Ciencias y últimamente se está asistiendo al surgimiento de instituciones específicas dedicadas a la investigación y docencia en el dominio de la Didáctica de las Ciencias. Como ejemplos citaremos el surgimiento de los Centres for Science and Mathematics Education en USA. (Streitz 1979) y en el Reino Unido (Richmond 1981), los Laboratoires Interuniversitaires de Recherche dans l'Enseignement des Sciences Physiques et Technologie (LIRESPT) en Francia (Tiberghien 1983) o los Centros di Studi per la Didattica en Italia (Bonera 1982). Esta íntima conexión entre las tareas de investigación educativa y la formación del profesorado y, a su vez, la existente entre esta última formación y la evaluación y mejora del sistema educativo tiene que hacer ver a las Administraciones de los distintos países que esta investigación debe ser parte importante en sus políticas educativas (Debeauvais 1989). 


\section{REFERENCIAS BIBLIOGRÁFICAS}

BLACKBURN V. y MOISAN C., 1986. The in-service training of teachers in the twelve member states of the European Community. (Presses Interuniversitaires Europeennes: Maestricht).

BLASCOR., 1989. La formación del profesoradoen Inglaterra: una visita de estudio, Revista Interuniversitaria de Formación del Profesorado, 4, pp. $111-128$.

BOLAM R., 1986. Interim Report-1978, en Hopkins D. (ed.), de Inservice Training Educational Development: an International Survey. (Croom Helm: London).

CALDERHEAD J., 1986. La mejora de la práctica de clase: aplicaciones de la investigación sobre toma de decisiones en la formación del profesorado, en Actas del I Congreso Internacional sobre Pensamiento de los Profesores y Toma de Decisiones. (Servicio de Publicaciones de la Unjversidad de Sevilla).

CALDERHEAD I., 1989. Reflective teaching and Teacher Education, Teaching \& Teacher Education, 5(1), pp. 43-51.

C.A.T.E., 1985. The Council's Approach To Accreditation, C.A.T.E. Note. (HMSO: London).

CONANT J., 1963. The education of americanteachers. (McGrawHill: New York).

DEBEAUVAIS M., 1989. Perspectivas, Vol. XIX (1).

D.E.S., 1983. Teaching in schools: the content of initial training. H.M.I. Discussion Paper. (HMSO: London).

D.E.S., 1984. Circular 3/84. Initial Teacher Training: Approval of courses. (HMSO: London).

DUARTE M. C. y FONTES M.A., 1989. Professor de Cincias, ¿qué perfil?, Enseñanza de las Ciencias, núm. Extra, Tomo 1, pp. 23-24.

FURIO C. y GIL D., 1984. Els Cursos d'Aptitud Pedagógica en quiestio, Estudi, octubre, pp. 12-13.

FURIO C. y GIL D., 1989. La didáctica de las Ciencias en la formación inicial del profesorado: una orientación y un programa teoricamente fundamentados, Enseñanza de las Ciencias, 7(3), pp. 257-265.

FURIO C., GIL D, y SENENT F., 1988. La formación del profesorado: introducción de estudios universitarios de didáctica de las disciplinas (ponencia presentada en las Jornadas sobre Formación Inicial del Profesoradode EEMM organizadas por el ICE de la Universidad de Alicante, 9-10 de junio). (Documento policopiado).

GUTIERREZ I., 1980. La formacion del Profesorado en los Congresos esparioles de Pedagogía, en Actas del VII Congreso Nacional de Pedagogía. (Sociedad Española de Pedagogía. Madrid).

HEWSON P.W. y HEWSON M., 1987. Science Teachers conception of teaching: Implications for teachers education, International Journal of Science Education, 9 (4), pp. 425440.
HEWSON P.W. y HEWSON M., 1988. On appropiate conception of teaching science: a view from studies of science learning, Science Education, 72(5), pp. 597-614.

IMBERNÓNF., 1990. La formación del profesorado, Cuadernos de Pedagogía, 178, pp. 88-97.

LAWSON A.E., COSTENSON K. y CISNEROS R., 1986. A summary of research in Science Education, Science Education, 70(3), pp. 19:-346.

MORENO A., 1990. La formación de los profesores de Ciencias en España: historia de una frustración, Revista Española de Física, 4(1), pp. 77-84.

PATRICK H., BERNBAUM G. y REID K., 1982. The structure and process of initial teacher education within universities in England and Wales. (University of Leicester. School of Education) (D.E.S.: London).

PENICK J.E. y YAGER R.E., 1986. Trends in Science Education: some obervations of exemplary programmes in the United States, European Journal of Science Education, 8(1), pp. $1-8$.

PESSOA DE CARVALHO A.M. y VIANNA D.M., 1988. A licenciatura em questão, Ciencia e Cultura, 40(2), pp. 143147.

RICHMOND P.E., 1981. Teaching and research in Science Education at Southampton University, European Journal of Science Education, 3(3), pp. 351-354.

ROYAL COMMISSION, 1888. Royal Commission on the elementary education acts. England and Walles. Final Report (Cross Report). (HMSO: London).

SCHÖN D. 1983. The reflective practitioner. (Temple Smith: London).

STENHOUSE L., 1975. An introduction to curriculum research development. (Heinemann: London).

STREITZ N.A., 1979. A graduate programme in Science Education: experiences and suggestions, European Journal of Science Education, 1(1), pp. 307-313.

TIBERGHIEN A., 1983. La investigación en un Laboratorio de Didáctica de las Ciencias Físicas, Enseñanza de las Ciencias, $\rfloor(3)$, pp. $187-192$.

TIBERGHIEN A., 1985. Quelques élements sur l'evolution de la recherche en didactique de la Physique, Revue Francaise de Pédagogie, 72, pp. 71-86.

TOBIN K. y ESPINET M., 1989. Impediments to change: applications of coaching in high school science teaching, Journal of Research in Science Teaching, 26(2), pp. 105120.

VERMA G.K. y BEARD R.M., 1981. What is educational research. (Gower: London).

ZEICHNER K.M., 1983. Alternatives paradigms of teacher education, Journal of Teacher Education, 34(3), pp. 3-9. 